hydrogen bonds to ring $\mathrm{V}$ carbonyl of the other Chl. As ROH, RNH, RSH compounds also form dimers this presents the intriguing idea that the in vivo dimer may be bound to similar groups in a protein but at present no experiments have been reported to test this idea.

Perhaps the most interesting observation on $\mathbf{P}_{700}$ is a transient electron proton resonance (EPR) emission spectrum produced by a single light flash on chloroplasts at room temperature. (Blankenship, et al., Proc. natn. Acad. Sci. U.S.A., 72, 4943; 1975). The EPR signal is thcught to be that of the primary acceptor as it does not correspond to $\mathrm{P}_{700}{ }^{+}$or a ferredoxin.

The emission is caused by the two spin states, normally thermally populated to $0.1 \%$ of one another, obtaining a significant population difference as a result of a particular chemical reaction favouring one state over the other. This phenomenon is called chemically induced dynamic electron polarisation (CIDEP). Immediately after the radical anion of the acceptor is formed the spin states still have the same populations as the anion's precursor and a CIDEP signal is observed until spin lattice relaxation equalises the spin populations. A triplet state of $\mathrm{Chl}$ is thought to be responsible for the emission from photosystem $I$, as a radical pair, which can also generate CIDEP are unlikely to diffuse together in the reaction centre.

There is still much controversy about the identity of the primary acceptor for $\mathbf{P}_{700}$. Malkin et al. (Biochim. biophys. Acta, 430, 399; 1976) maintain that the acceptor is a ferredoxin, but McIntosh and Bolton (Biochim. biophys. Acta., 430, 555; 1976) and Evans et al., (Nature, 256, 668; 1975) dispute this and claim that a species with ESR signal $g=1.76$ is the primary acceptor and that ferredoxin has a secondary role. The relationship of the signal seen by Blankenship to these acceptor signals is not known. The evidence is not as complete as with the bacterial reaction centre but the initial charge separation in photosystem I appears to produce a $(\mathrm{Chl})_{2}{ }^{+}$species and an unidentified radical anion before the ferredoxin is reduced.

Much less is known of photosystem II reaction centres, $\mathbf{P}_{680}$, than of $\mathbf{P}_{700}$, although there is good evidence that $\mathbf{P}_{680}$ is the primary donor, possibly a chlorophyll dimer (Pulles, et al., Biochim. biophys. Acta., 440, 98; 1976). The observation, by McIntosh and Bolton, of a CIDEP emission from $\mathrm{Chl}$ in photosystem II is thus of particular interest as this signal can only arise from a Chl triplet state at the temperatures used. They suggest that the triplet state forms very rapidly from the singlet by the formation of a Chl ${ }^{+}$
$\mathrm{Chl}^{-}$species. The primary acceptor would then have to be adjacent to the dimer in order to accept the electron at a rate much faster than the charge recombination for efficient charge separation to occur.

Many similarities between reaction centres are now emerging. All involve chlorophyll dimers; at least two, and perhaps all, contain short lived "transient" acceptors between the dimer and the "primary" acceptor which is perhaps a device to prevent rapid charge recombination of the ion pair. Although both $P_{700}$ and $P_{680}$ appear to involve triplet states formed, perhaps very quickly, after excitation of the dimer species the reasons for preferring triplets over singlets are unknown. Consequently picosecond spectroscopy will be invaluable in observing transients produced in the initial processes and our present knowledge of reaction centres should provide impetus to produce model systems to mimic photosynthetic processes.

\section{Homostable operons?}

\section{from E. G. Richards}

READERs who glance through the pages of the more theoretical journals will be aware of the immense activity over the past 15 years on the statistical mechanical basis of the melting of DNA. Certainly every well-informed undergraduate will know that DNA undergoes a cooperative helix coil transition when heated that gives rise to a sharp melting curve. However theory suggests that things are not quite that simple. For instance, a hypothetical DNA molecule consisting of a region of 1,000 A-T base pairs followed by a similar one of $\mathrm{G}-\mathrm{C}$ pairs would give a melting curve consisting of two sharp steps. To produce a sharp step, theory suggests, requires a 'homostable' region of 500 or more base pairs with no sequences of more than a few adjacent $A-T$ or adjacent $\mathrm{G}-\mathrm{C}$ base pairs. A DNA molecule consisting of several such homostable regions would then melt in a series of sharp steps, and the melting temperature of each step should reflect the base composition of the corresponding region.

Another and more informative way of depicting melting behaviour is by the differentiated melting curve in which sharp steps now appear as sharp peaks. In recent years much expertise and ingenuity has been expended in constructing equipment capable of producing such curves at high resolution so that the fine structure of a melting curve occurring over $10^{\circ} \mathrm{C}$ or so could be resolved into a dozen or more sharp peaks each a degree or so in width.
Wada and Tachibana, in a paper in Nature $(\mathbf{2 6 3}, 439 ;$ 1976) note that short DNA species do indeed manifest such fine structure in their differentiated melting curves and, moreover, that the melting temperature of the individual peaks does indeed bear the expected relation to the base composition of the corresponding DNA region as revealed by spectral analysis. In longer DNA species, presumably consisting of many homostable regions with closely similar stabilities, the fine structure is less apparent.

These authors then go on to consider the behaviour in more detail of a short DNA (fd phage, 6,000 base pairs) and fragments produced from it by the restriction enzyme RHinHI.

First, the intact DNA (RF linear) is shown to have a differentiated melting curve consisting of more than a dozen sharp peaks distributed over a $10{ }^{\circ} \mathrm{C}$ melting range. The product formed by the action of the restriction enzyme and consisting of three fragments gives a broadly similar curve. When the three fragments were separated by polyacrylamide gel electrophoresis they individually gave curves with considerably fewer peaks. Nevertheless, when the three curves corresponding to the three fragments were summed, the result was closely similar to the curve of the mixture.

These results suggest that the peaks in the differentiated melting curves arise from homostable regions situated at specific locations in the molecule. It would appear from the relative sizes of the peaks and from the fact that splitting is observed that some peaks correspond to several homostable regions with closely similar stabilities.

A detailed comparison of the curves for the intact molecule and for the mixture of its fragments suggests that one homostable region may affect the melting temperature of others so that peaks in one of the curves may be split in the other by moving to slightly different temperatures. It is tempting to speculate that such interactions are between adjacent homostable regions, in which case it might be possible to map the regions along the molecule. Certainly the effect presents interesting statistical mechanical problems.

Wada and Tachibana end by speculating as to whether these homostable regions have some biological role. The data suggest that each operon can contain only a few and perhaps only one homostable region, and the authors suggest that the regions might play some part in recombination. They also note that wobble interactions in translation could well mean that homostable regions and active folypeptide sequences could be selected for simultaneously. 